
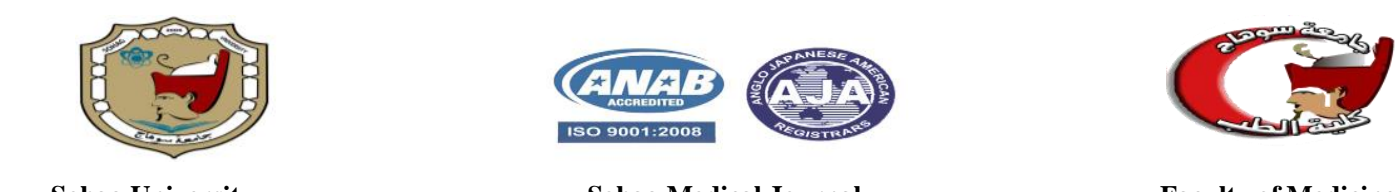

\title{
Comparative study between fluoroscopic-guided and ultrasound- guided genicular nerve pulsed radiofrequency neurotomy for painful knee osteoarthritis
}

\author{
Wael Alham Mahmoud, Ahmed Talaat Mohamed, Ahmed Elsaied Abdelrahman, Fawzy \\ Abbas Badawy
}

Department of anesthesia, faculty of medicine, sohag university

\begin{abstract}
Introduction: Knee osteoarthritis (OA) has become a worldwide health issue. Genicular nerve ablation has been used for the management of pain in knee osteoarthritis patients. This study aims to compare between fluoroscopy and ultrasound-guided genicular ablation. Patients and methods: A total of 30 cases were included, after approval of the ethics committee at sohag university, and they were divided into two groups, 15 cases underwent the fluoroscopic guided procedure, while the other 15 cases underwent the ultrasoundguided procedure. Both VAS and WOMAC scores were assessed and recorded in all cases after 1-, 3-, and 6-month follow-up visits.

Results: Patient demographics and characters did not differ significantly between the two groups. Both groups showed a marked improvement of both scores when compared to their basal score. However, the ultrasound group showed a marked improvement compared to fluoroscopy.

Conclusion: Based on our study findings, we believe that ultrasound-guided genicular verve ablation provides better results compared to the fluoroscopic guided procedure. However, fluoroscopy still provides an effective guidance method.

Keywords: Genicular ablation, osteoarthritis, fluoroscopy, ultrasound.
\end{abstract}

\section{Introduction}

One of the most important worldwide health problems is Knee osteoarthritis (OA). Increasing the prevalence of knee $\mathrm{OA}$ is associated with the aging of the population and the increasing prevalence of obesity (1). It has an estimated prevalence of $24 \%(2,3)$.

Knee OA can be treated with several treatment modalities including non-pharmacological methods (education, exercising, and electrotherapy) pharmacological therapies (analgesics, topical agents, and chondroprotective drugs), non-surgical procedures (intra-articular steroid and viscosupplementation, platelet-rich plasma, periosteal stimulation therapy, acupuncture), and surgical interventions (arthroscopy, arthroplasty). Nevertheless, patients with knee OA may not feel adequate pain control despite all previous treatment modalities (4).

One of the methods of pain relief is radiofrequency (RF) neurotomy and may be used to improve functioning by destroying nerves innervating painful tissue or by disturbing the transmission of pain signals (5). Many other conditions can be treated with radiofrequency neurotomy 
including trigeminal neuralgia, cancer, and spinal pain (6).

Patients unresponsive to conventional treatment and inoperable patients due to comorbidities and/or old age are good candidates for radiofrequency neurotomy as it helped manage pain-related knee OA in those patients (7).

Obturator, femoral, saphenous, common peroneal, and tibial nerves are the genicular nerves that supply the knee region. Management of chronic knee pain related to $\mathrm{OA}$ can be done with genicular nerve RF neurotomy, it is considered a reliable method in its management (5).

The use of imaging devices in the performance of nerve blocks can help to increase procedural accuracy and reducing complications compared to a blind technique. Therefore, selecting the appropriate imaging device is imperative for increasing the success rate of a nerve block and reduce complications. $(8,9)$.

This study was conducted to compare between fluoroscopy and ultrasoundguided genicular nerve pulsed RF treatment on chronic knee pain and function in patients with knee osteoarthritis.

\section{Patients and methods Study design}

This is a prospective randomized study that was conducted at Sohag University Hospitals during the period between 20172020 , after approval of the ethics committee at sohag university,

\section{Study cases}

A total of 30 cases $(n=30)$ diagnosed with knee osteoarthritis were included. They were randomly divided into two equal groups, randomization was done using a computer program, the first included cases who underwent fluoroscopic guided genicular ablation, and the other one included the other 30 cases who underwent the ultrasound-guided procedure.

\section{Inclusion criteria}

The American College of Rheumatology (ACR) recommendations for the diagnosis of OA was used in this study (10). Patients with stage 2 or higher OA-related radiological changes based on the Kallgren-Lawrence (K/L) rating scale were included in the study (11).

Additional clinical criteria for inclusion in the study were pain at least moderate in severity or pain daily for more than 3 months and patients have to be clinically unresponsive to conservative treatment modalities (physical therapy and rehabilitation practices, orally administered analgesics and anti-inflammatory agents) and unfit or refuse to have arthroplasty.

All patients underwent diagnostic genicular nerve blocks with a local anesthetic, which was performed under fluoroscopic guidance. The targets included the superior lateral SL, superior medial SM, and inferior lateral IM genicular nerves. Lidocaine ( $2 \mathrm{~mL}$ of $2 \%$ ) was injected at each target site. Responses were recorded as positive if the patient experiences a decrease in numeric pain scores of at least $50 \%$ for more than $24 \mathrm{~h}$. Patients with a positive response were included in the RF neurotomy procedures.

\section{Exclusion criteria}

Patients with previous knee surgeries, infection on the site of injection, acute knee pain, serious neurological or psychiatric conditions, connective tissue disorders, or coagulopathy were excluded from our study.

\section{Outcomes}

The degree of pain improvement was assessed via VAS (visual analog score), 
while the function was assessed via the WOMAC score (Western Ontario and McMaster Universities Arthritis Index).

\section{Patient consent}

Informed written consent was obtained from all cases before the procedure.

The advantages and drawbacks of every approach were explained to them.

Besides, the study was approved by the local ethical committee.

\section{Ultrasound-guided procedure}

Ultrasound scanning of the knees was performed using a $12-5 \mathrm{MHz}$ linear transducer. During the examination, the ultrasound probe was placed sagittally in the medial aspect of the knee in full extension with the patient lying on the lateral side. Thus, the anatomic landmarks of the patients were imaged. The identification of the SL, SM, and IM genicular nerves using ultrasound was performed.

Lower femoral and upper tibial regions were sterilely prepped. Anesthesia was provided by subcutaneous $2 \%$ lidocaine injections. A 10-cm, 22-gauge RF thermocoagulation cannula with a $10 \mathrm{~mm}$ active tip was placed for each genicular nerve. Anterior-posterior and late-ral fluoroscopic images were obtained to confirm the position of the RF thermocoagulation cannula tip. The RF probe was placed perpendicular to the presumed length of the nerve.

A $50 \mathrm{~Hz}$-frequency sensorial stimulation was applied with a threshold of

$<0.6 \mathrm{~V}$. During the sensorial stimulation, the patients were asked if they felt a tingling, pain, or discomfort inside the knee. The RF probe was maintained in place until one of those feelings were elicited. Also, $2.0 \mathrm{~V}$ motor stimulation was applied at a frequency of $2 \mathrm{~Hz}$ to determine the absence of fasciculation.
Before the activation of the RF generator, an injection of $2 \mathrm{ml} \mathrm{1 \%}$ lidocaine was applied.

Subsequently, RF lesions were generated by applying pulsed RF treatment to the SL, SM, and IM genicular nerves for 120 seconds twice at $42^{\circ} \mathrm{C}$.

\section{Fluoroscopic guided procedure}

Patients were placed in the supine position and their knee was supported by a small pillow placed beneath the popliteal fossa. Fluoroscopic images of the tibiofemoral joint were obtained. Possible locateons of genicular nerves were determined on the lateral and medial aspects of the lower end of the femoral bone, on the medial aspect of the tibia, under fluoroscopic guidance.

\section{Follow up}

Both VAS and WOMAC scores were assessed and recorded in all cases after 1-, 3-, and 6-month follow-up visits.

\section{Statistical analysis}

Statistical analysis was performed using the SPSS software program. Data were presented as mean \pm standard deviation, or number (\%). A comparison between both groups was performed by an independent t-test or chi-squared test as appropriate. Changes were evaluated using the repeated measures general linear model. A value of $a P$ value less than 0.05 was considered statistically significant.

\section{Results}

The mean age of the fluoroscopy group was 55.9 years, while it was 54.6 years for the ultrasound group. Females were more predominant than males in both groups $(73 \%$ and $93 \%$ respectively). Other data are shown in table (1) 
SOHAG MEDICAL JOURNAL

Vol. 25 No. 1 Jan 2021

\begin{tabular}{|c|c|c|c|c|c|}
\hline \multicolumn{2}{|l|}{ Variables } & \multirow{2}{*}{$\begin{array}{c}\begin{array}{c}\text { Fluoroscopy } \\
\text { group }(n= \\
15)\end{array} \\
55.9 \pm 6.9\end{array}$} & \multirow{2}{*}{$\begin{array}{c}\text { Ultrasound } \\
\text { group }(n= \\
15) \\
54.6 \pm 6.5\end{array}$} & \multirow{2}{*}{$\begin{array}{c}\begin{array}{c}\text { Difference } \\
(95 \% \text { CI })\end{array} \\
-3.7,6.3\end{array}$} & \multirow{2}{*}{$\begin{array}{c}\text { P-value } \\
0.61 \\
\end{array}$} \\
\hline Age & Mean \pm SD & & & & \\
\hline BMI & Mean \pm SD & $28.9 \pm 2.6$ & $29 \pm 2$ & $-1.8,1.7$ & 0.94 \\
\hline \multirow[t]{2}{*}{ Sex } & Male & $27 \%(4)$ & $7 \%(1)$ & \multirow[t]{2}{*}{$-0.36,0.76$} & \multirow[t]{2}{*}{0.33} \\
\hline & Female & $73 \%(11)$ & $93 \%(14)$ & & \\
\hline \multirow[t]{2}{*}{ ASA } & I & $93 \%(14)$ & $87 \%(13)$ & \multirow[t]{2}{*}{$-1.7,1.8$} & \multirow[t]{2}{*}{1} \\
\hline & II & $7 \%(1)$ & $13 \%(2)$ & & \\
\hline \multirow{3}{*}{$\begin{array}{l}\text { Osteoarth } \\
\text { ritis }\end{array}$} & II & $47 \%(7)$ & $40 \%(6)$ & \multirow[t]{3}{*}{$-1.2,1.3$} & \multirow[t]{3}{*}{0.55} \\
\hline & III & $53 \%(8)$ & $47 \%(7)$ & & \\
\hline & IV & $0 \%$ & $13 \%(2)$ & & \\
\hline \multirow[t]{2}{*}{ Site } & Right & $67 \%(10)$ & $53 \%(8)$ & \multirow[t]{2}{*}{$-1.3,1.6$} & \multirow[t]{2}{*}{0.71} \\
\hline & Left & $33 \%(5)$ & $47 \%(7)$ & & \\
\hline
\end{tabular}

Table (1): Patient characteristics. -Data are expressed as mean $\pm \mathrm{SD}$ or percentage and number.

-P-value is significant when $<0.05$.
On the assessment of the VAS score in both groups, it was evident that there was a marked improvement of pain on the follow-up visits when compared to pre-intervention scores $(\mathrm{p}<0.05)$. Tables (2 and 3 ) illustrate these data.
Although the VAS score did not differ before or 1-month after the intervention, the ultrasound group showed better scores on the two subsequent visits ( $\mathrm{p}=0.014$ and 0.17 respectively). Table (4) illustrates these data.

\begin{tabular}{|l|c|c|c|c|c|}
\hline \multicolumn{2}{|l|}{ VAS } & $\begin{array}{c}\text { Fluoroscopy } \\
\text { group }(n=15)\end{array}$ & $\begin{array}{c}\text { Ultrasound } \\
\text { group }(n=15)\end{array}$ & $\begin{array}{c}\text { Difference } \\
(95 \% \text { CI })\end{array}$ & $\begin{array}{l}\text { P- } \\
\text { value }\end{array}$ \\
\hline Basal & Mean \pm SD & $7.5 \pm 0.7$ & $7.3 \pm 0.6$ & $-0.29,0.69$ & 0.41 \\
\hline One month & Mean \pm SD & $5.3 \pm 0.6$ & $5.2 \pm 0.8$ & $-0.37,0.64$ & 0.59 \\
\hline Three months & Mean \pm SD & $5.3 \pm 0.6$ & $4.4 \pm 1.3$ & $0.2,1.67$ & 0.014 \\
\hline Six months & Mean \pm SD & $6.0 \pm 1$ & $4.7 \pm 1.8$ & $0.24,2.3$ & 0.017 \\
\hline
\end{tabular}

Table (2): Basal and follow-up levels of the VAS score of both groups.

-Data are expressed as mean $\pm S D$.

$-P$-value is significant when $<0.05$.

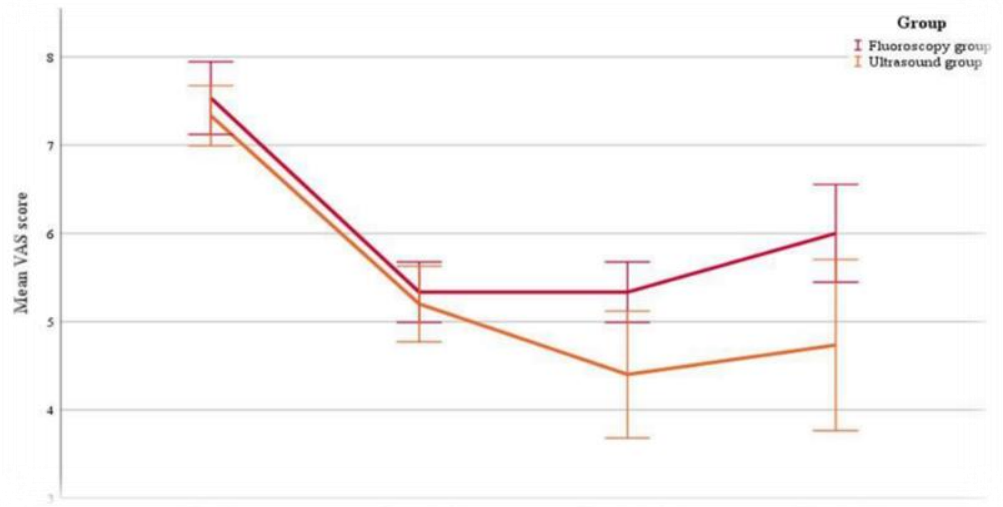

Figure (1): Changes of VAS scores before and after intervention in both study groups 
WOMAC score showed a significant improvement in both groups during the scheduled follow up visits $(\mathrm{p}<0.005)$. These data are shown in table (5 and 6). When comparing the WOMAC score in both groups, the ultrasound group

\begin{tabular}{|l|l|c|c|c|c|}
\hline \multicolumn{2}{|l|}{ WOMAC } & $\begin{array}{c}\text { Fluoroscopy } \\
\text { group } \\
(\mathbf{n = 1 5})\end{array}$ & $\begin{array}{c}\text { Ultrasound } \\
\text { group } \\
(\mathrm{n}=15)\end{array}$ & $\begin{array}{c}\text { Difference } \\
(\mathbf{9 5 \%} \text { CI })\end{array}$ & P-value \\
\hline Basal & Mean \pm SD & $\mathbf{6 7 . 7} \pm 6.6$ & $\mathbf{6 8 . 5} \pm \mathbf{5 . 6}$ & $\mathbf{- 5 . 2 , 3 . 5}$ & $\mathbf{0 . 6 9}$ \\
\hline One month & Mean \pm SD & $\mathbf{6 2 . 2} \pm 5.9$ & $\mathbf{5 5 . 7} \pm 6.8$ & $\mathbf{1 . 9 , 1 1 . 1}$ & $\mathbf{0 . 0 0 7}$ \\
\hline Three months & Mean \pm SD & $\mathbf{5 9 . 3} \pm 5.5$ & $\mathbf{5 0 . 8} \pm 7$ & $\mathbf{3 . 9 , 1 3}$ & $\mathbf{0 . 0 0 1}$ \\
\hline Six months & Mean \pm SD & $\mathbf{6 1 . 1} \pm 5$ & $\mathbf{5 3 . 3} \pm 9.4$ & $\mathbf{2 . 4 , 1 3 . 2}$ & $\mathbf{0 . 0 0 6}$ \\
\hline
\end{tabular}

Table (3): Basal and follow-up levels of the WOMAC score of both groups

-Data are expressed as mean $\pm \mathrm{SD}$.

-P-value is significant when $<0.05$.

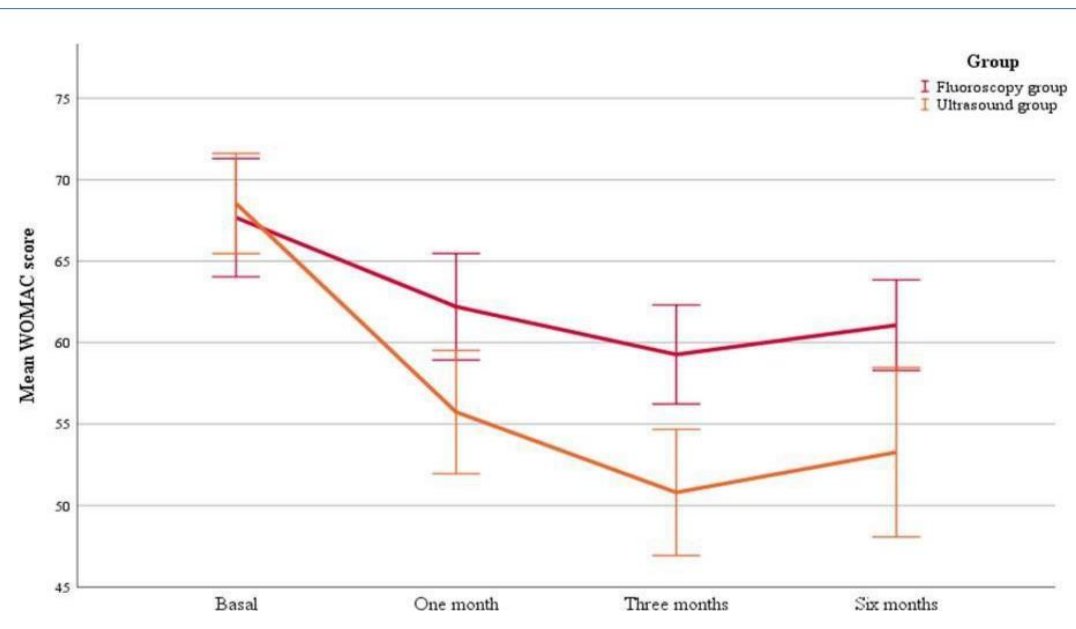

Figure (2): Changes in WOMAC score before and after intervention in both groups.

\section{Discussion}

The knee joint is innervated by articular branches of femoral, common peroneal, saphenous, tibial, and obturator nerves. The RFA of these nerves had been attempted for the last few years for relief of pain from knee osteoarthritis (12). Several studies have compared the effecttiveeness of fluoroscopy- vs ultrasoundguided blocks for chronic pain management. Nevertheless, studies handling the comparison between ultrasound and fluoroscopy in genicular nerve ablation are few in the current literature (13).
However, whether one imaging method is superior to the other remains unclear. Fluoroscopic guidance has several advantages for genicular nerve blocks. First, the superolateral, superomedial, and inferomedial genicular nerves traverse along the periosteal areas connecting the shaft to the epicondyle (5).

Therefore, a fluoroscopic view of the knee joint can easily identify target points for radiofrequency ablation of genicular nerves. Second, ultrasound does not provide a clear visualization of smaller-gauge 
needles at deep tissue levels, whereas fluoroscopic imaging provides good needle visualization regardless of tissue depth and needle gauge. Third, the use of real-time contrast fluoroscopy and digital subtraction angiography can prevent unintentional intravascular injection (14). The limitation of this technique is that only the ablation of the three genicular nerves was possible and recent studies had also shown that there are chances of injury to the vessels which accompany these nerves (15). Conversely, it was reported that ultrasound enables the physician to ablate the seven genicular nerves (12).

On the other hand, ultrasound guidance during genicular nerve blocks offers unique advantages over fluoroscopy. First, neither the patients nor clinicians are exposed to radiation during an ultrasound-guided procedure. This is deemed as the best advantage ultrasound has over fluoroscopy. Second, ultrasound can provide a real-time image of soft tissues (nerves, muscles, vessels, etc.), an image of needle tip advancement relevant to surrounding structures, and visualizeation of injectate spread (16).

Third, it can both statically and dynamically guide diagnostic and therapeutic procedures. Another advantage of ultraso-und-guided genicular nerve blocks includes the visualization of genicular arteries and even the occasional identification of genicular nerves, which may increase genicular nerve blockade precision (13).

However, differences in surgical proficiency and patient obesity may hinder the selection of ultrasound $(17,18)$.

This study was conducted at the Outpatient pain clinic of Sohag University hospital aiming to compare fluoroscopy and ultrasound-guided genicular nerve pulsed $\mathrm{RF}$ treatment on chronic knee pain and function in patients with knee osteoarthritis.

A total of 30 cases were included and they were divided into two equal groups; the first group included 15 cases who underwent fluoroscopy-guided genicular nerve ablation, and the other group included the remaining 15 cases who underwent ultrasound-guided ablation. In the current study, the mean age of the included cases was 55.9 and 54.6 years for both groups respectively. There was no statistically significant difference between the two groups regarding patients' age $(\mathrm{p}=0.61)$.

Another recent study included cases with a mean age of 66.8 and 65.2 years for the fluoroscopy and ultrasound groups respectively. There was no

difference between the two groups regarding patient age $(\mathrm{p}=0.544)$ and this comes in line with our study (13).

In our study, the fluoroscopy group included 4 males (27\%) and 11 females (73\%), whereas the ultrasound group included 1 male (7\%) and 14 females (93\%). Gender also did not constitute a significant parameter between the study groups $(\mathrm{p}=0.33)$.

In another study, female sex was also predominant. The fluoroscopic group included 20 females $(80 \%)$ and 5 males (20\%) while the ultrasound group included 24 females (96\%) and 1 male (4\%). Like our study, no significance was found between the two groups regarding patient sex (19).

Regarding the BMI of the included cases in our study, the mean BMI was

28.9 and $29.0 \mathrm{~kg} / \mathrm{m}^{2}$ for both groups respectively. Like age and sex, BMI was not significantly different between the two study groups $(\mathrm{p}=0.94)$.

Doo-Hwan Kim and his colleagues also included cases in the two groups without a significant difference in BMI $(\mathrm{p}=$ 
$0.071)$. The mean values of BMI of the included cases were 24.1 and $25.8 \mathrm{~kg} / \mathrm{m}^{2}$ for fluoroscopy and ultrasound groups respectively (13). Sar1 et al. included cases with a mean BMI of 29.45 and 30.94 for both groups respectively ( $p>$ 0.05) (19).

In our study, the grade of osteoarthritis did not differ significantly between the two groups $(\mathrm{p}=0.55)$. The fluoroscopy group included $47 \%$ and $53 \%$ of its cases had osteoarthritis classes II and III respectively. The ultrasound group included classes II, III, and IV with a percentage of 40,47 , and $13 \%$ respectively.

In another study comparing the two groups, grades of knee osteoarthritis did not differ significantly between the two groups, and that copes with our study findings. The Fluoroscopy group included cases with a percentage of $64.3 \%, 32.1 \%$, and $3.6 \%$ for grades II, III, and IV in the fluoroscopy group. Besides, the ultrasound group included the same grades with a percentage of $43.3 \%, 43.3 \%$, and $13.3 \%$ respectively (13).

Regarding the affected side in the current study, it was not significant between the two groups $(p=0.71)$. The cases in the fluoroscopy group were having affected the right knee in $67 \%$ of cases, while the left knee was affected in $33 \%$ of cases. On the other hand, the right knee was affected by $53 \%$ while the left knee was affected by $47 \%$ of ultrasound cases.

Doo-Hwan Kim and his colleagues included fluoroscopy cases with a rightsided disease (30\%), left-sided $(36.7 \%)$, and bilateral disease (33.3\%). In the other group, $35.5 \%$ were having bilateral disease, $32.3 \%$ had right-sided affection, and the same ratio had the left-sided disease (13).

Significant improvement in pain and satisfaction in the RF treatment group described by Choi et al and support fluoroscopically guided RF neurotomy of the sensory nerves (GNs) supplying the knee joint (20).

An Egyptian study assessed the efficacy of fluoroscopy-guided genicular ablation compared to medical treatment. The authors found that VAS scores significantly decreased from 7.07 at baseline, down to $2.47,2.83$, and 3.13 at 2-week, 3-, and 6-month follow-up visits respectiveely. It was evident that fluoroscopyguided ablation was significantly better than medications regarding pain relief overall follow up visits (21).

This also coincides with Masala et al, who concluded that the early decreasing of mean VAS scores by the end of 1st week after pulsed RF, with marked pain relief in the following months in 40 patients suffering from knee OA unresponsive to conservative therapy (22).

When it comes to the ultrasound group in our study, it was evident that the included cases experienced a marked reduction of their pain at the scheduled follow up visits. VAS score had a mean of 7.3 before the intervention, which decreased down to 5.2, 4.4, and 4.7 at 1-, 3-, and 6month follow-up visits respectively $(\mathrm{p}<$ $0.0005)$.

In another study that assessed the ultrasound-guided genicular nerve ablation, significant improvement in the pain intensity at rest, on movement, and on walking for up to 6 months (p-value $<0.05$ )in the RF treatment group. At 1 month, almost all patients had more than $50 \%$ improvement in pain intensity at rest, on movement, and on walking. Interestingly, even at 6 months, eight, five, and three patients had more than $50 \%$ relief in pain intensity on rest, on movement, and on walking respectively (12).

Another study also confirmed the efficacy of ultrasound-guided genicular nerve ablation in patients with or without 
previous knee operations for degenerative diseases. VAS scores decreased significantly from 8.2 (before intervention), down to 2.8 after 3 weeks, and 3.2 after 3 months $(\mathrm{p}<0.01)$ in the non-operated group. The same significance was also detected in the operated group as the VAS score decreased from 8.5 preintervention down to 4.5 and 4.7 at the same follow up visits (23).

When comparing both groups regarding VAS scores, although no difference was detected at baseline nor after 1 month, the ultrasound group had a marked pain reduction when compared to the fluoroscopy group at 3-, and 6-month follow-up visits ( $\mathrm{p}=0.014$ and 0.017 respectively).

Another study found no difference in efficacy between these imaging methods for GNB. This similarity between both image guidance methods for GNB may be due to the anatomical properties of genicular nerves. Genicular nerves travel along each genicular artery (13). These results contradict our results.

On the assessment of the WOMAC score in the fluoroscopy group, it showed a significant decrease at the follow-up visits. From a baseline of 67.6, it decreased down to $62.2,59.3$, and 61.1 after 1,3 , and 6 months respectively $(\mathrm{p}<0.05)$.

The previously mentioned study also found that fluoroscopy was successful in reducing WOMAC score from a baseline of 93.53 down to $21.67,24.23$, and 33.13 at 2-week, 3-, and 6-month follow-up visits. However, WOMAC scores did not differ significantly from the medically treated group except at a 6-month follow up visit (21).

Regarding the ultrasound group in our study, the WOMAC score decreased significantly from a baseline of 58.5 down to 55.7, 50.8, and 53.3 at the same followup visits respectively $(\mathrm{p}<0.001)$.
Significant pain relief and functional improvement in severe to moderate knee degenerative osteoarthritis after the PRF procedure was reported by Kesikburun et al. (24).

Significant improvement of pain, stiffness, and physical function as measured by the WOMAC score was reported by Ahmed and Arora who found that it was improved significantly from $78.38 \pm 2.97$ before ultrasound-guided genicular ablateon to $38.38 \pm 5.82$ and $39.25 \pm 5.12$ at 1 and 6 months, respectively (p-value $<0.05)(12)$.

A previously mentioned study also detected a significant reduction of WOMAC score after ultrasound-guided nerve ablation ( $\mathrm{p}<0.01$ ). It decreased from 62.7 down to 33.8 after 3 weeks, and 37.3 after 3 months in cases without a history of previous knee operations. In the operated group, WOMAC significantly decreased from 65.1 down to 40.7 and 46.2 at the same follow-up periods respectively (23). When comparing both groups regarding the WOMAC score, although they did not differ significantly before the intervention, the ultrasound group showed its superiority when compared to the fluoroscopy group during the three scheduled follow-up visits $(p=0.007,0.001$, and 0.006 respectively).

Fluoroscopy and musculoskeletal ultrasound groups were compared by another study that found no significant difference observed between the two groups when pre- and post-treatment data were compared concerning pain and functional status (19).

Based on our study findings, it was evident that both radiological techniques were effective in pain reduction in knee osteoarthritis patients. Nevertheless, the ultrasound group was more superior regarding pain relief and functional improvement when compared to the other group. 
We believe that ultrasound may be more suitable for GNB. As mentioned previously, ultrasound has several advantages over fluoroscopy. Given that GNB or RFGN is usually repeated periodically due to its finite duration, cumulative doses of radiation may damage several other organs, including the skin, bones, thyroid glands, and lungs.

The main drawback of our study is the small sample size. Hence, more studies including more cases should be conducted in the future.

\section{Conclusion}

Based on our study findings, we believe that ultrasound-guided genicular verve ablation provides better results compared to the fluoroscopic guided procedure. However, fluoroscopy still provides an effective guidance method.

\section{References}

1. Neogi T. The epidemiology and impact of pain in osteoarthritis. Osteoarthritis Cartilage. 2013;21(9):1145-53.

2. Hunter DJ, McDougall JJ, Keefe FJ. The symptoms of osteoarthritis and the genesis of pain. Rheum Dis Clin North Am. 200-8;34(3):623-43.

3. Pereira D, Peleteiro B, Araujo J, Branco J, Santos RA, Ramos E. The effect of osteoart-hritis definition on prevalence and incidence estimates a systematic review. Osteoarthritis Cartilage. 2011;19(11):1270-85.

4. Sarı S, Aydın ON, Turan Y, Özlülerden P, Efe U, Kurt Ömürlü İ. Which one is more effective for the clinical treatment of chronic pain in knee osteoarthritis: radiofrequency neurotomy of the genicular nerves or intr-aarticular injection? International journal of rheumatic diseases. 2018;21(10):1772-8.

5. Choi WJ, Hwang SJ, Song JG, et al. Radiofrequency treatment relieves chronic knee osteoarthritis pain: a double- blind rand-omized controlled trial. Pain. 2011;15-2(3):481-7.

6. Masala S, Fiori R, Raguso M, Morini M, Calabria E, Simonetti G. Pulse-dose radiofre-quency for knee osteoarthritis. Cardiovasc Intervent Radiol. 2014;37(2):482-7.

7. Protzman NM, Gyi J, Malhotra AD, Kooch JE. Examining the feasibility of radiofreq-uency treatment for chronic knee pain after total knee arthroplasty. PM R. 2014;6(4):373-6.

8. Ucuncu F, Capkin E, Karkucak M, et al. A comparison of the effectiveness of landmark-guided injections and ultrasonography-gui-ded injections for shoulder pain. The Clinical journal of pain. 2009;25(9):786-9.

9. Hoeber S, Aly A-R, Ashworth N, Rajaseka-ran S. Ultrasound-guided hip joint injections are more accurate than landmark-guided inje-ctions: a systematic review and meta-analysis. $\mathrm{Br} \mathrm{J}$ Sports Med. 2016;50(7):392-6.

10.Altman RD. Criteria for the classification of clinical osteoarthritis. J Rheumatol Suppl. 1991;27:10-2.

11. Kellgren JH, Lawrence JS. Radiological assessment of osteoarthritis. Ann Rheum Dis. 1957;16(4):494-502.

12. Ahmed A, Arora D. Ultrasound-guided radiofrequency ablation of genicular nerves of the knee for relief of intractable pain from knee osteoarthritis: a case series. British journal of pain. 2018;12(3):145-54.

13.Doo-Hwan Kim M, Myung-Su Lee M, Sookyung Lee M, Syn-Hae Yoon M. A Prospective Randomized Comparison of the Efficacy of Ultrasound-vs Fluoroscopy-Guided Genicular Nerve Block for Chronic Knee Osteoarthritis. Pain physician. 2019;22:139-46.

14.Baker R, Dreyfuss P, Mercer S, Bogduk N. Cervical transforaminal injection of corticosteroids into a radicular artery: a 
possible mechanism for spinal cord injury. PAIN®. 2003;103(1-2):211-5.

15.Le PU. Is genicular nerve radiofrequency ablation safe? A literature review and anatomical study. Pain Physician. 2016;19:E697-E705.

16. Narouze SN, Vydyanathan A, Kapural L, Sessler DI, Mekhail N. Ultrasoundguided cervical selective nerve root block: a fluoroscopy-controlled feasibility study. 2009.

17. Maarse W, Watts AC, Bain GI. Mediumterm outcome following intra-articular corticosteroid injection in first $\mathrm{CMC}$ joint arthritis using fluoroscopy. Hand Surgery. 2009;14(02n03):99-104.

18.Handling LE, Hiltunen OJ. Preoperative difficulties in fluoroscopy of the femoral head in the massive obese patient: enhanced visualization by the intraarticular contrast agent. Archives of orthopedic and trauma surgery. 2006;126(7):498-9.

19.Sarı S, Aydın ON, Turan Y, et al. Which imaging method should be used for genicular nerve radiofrequency thermocoagulation in chronic knee osteoarthritis? Journal of clinical monitoring and computing. 2017;31(4):797-803.

20.Choi W-J, Hwang S-J, Song J-G, et al. Radiofrequency treatment relieves chronic knee osteoarthritis pain: a double- blind randomized controlled trial. PAIN@. 2011;152(3):481-7.

21.Kamel EZ. Fluoroscopic guided radiofrequency of genicular nerves for pain alleviation in chronic knee osteoarthritis: a single-blind randomized controlled trial. Pain physician. 2018;21:169-77.

22. Masala S, Fiori R, Raguso M, Morini M, Calabria E, Simonetti G. Pulse-dose radiofrequency for knee osteoarthritis. Cardiovascular and interventional radiology. 2014;37(2):482-7.

23.Erdem Y, Sir E. The Efficacy of Ultrasound-Guided Pulsed Radiofrequency of Genicular Nerves in the Treatment of Chronic Knee Pain Due to Severe Degenerative Disease or Previous Total Knee Arthroplasty. Medical science monitor: international medical journal of experimental and clinical research. 2019;25:1857.

24.Kesikburun S, Yaşar E, Uran A, Adigüzel E, Yilmaz B. Ultrasound-guided genicular nerve pulsed radiofrequency treatment for painful knee osteoarthritis: A preliminary report. Pain Physician. 2016;19(5):E7519. 